\title{
A Slower Heart Rate and Therapeutic Hypothermia Unmasked Early Repolarization Syndrome in a Ventricular Fibrillation Survivor
}

\author{
Kanae Hasegawa, ${ }^{1}$ MD, Shinsuke Miyazaki, ${ }^{1}$ MD, Tetsuji Morishita, ${ }^{1}$ MD, Kenichi Kaseno, ${ }^{1}$ MD, \\ Kaori Hisazaki, ${ }^{1}$ MD, Naoki Amaya, ${ }^{1}$ MD, Hiroyasu Uzui, ${ }^{1}$ MD and Hiroshi Tada, ${ }^{1}$ MD
}

\begin{abstract}
Summary
Patients presenting with aborted cardiac arrest who display early repolarization generally are diagnosed with early repolarization syndrome. Therapeutic hypothermia is a standard strategy to improve neurological outcome in comatose patients after cardiac arrest. We present here a patient in whom therapeutic hypothermia exacerbated the J-wave amplitude and morphology, which resulted in episodes of refractory ventricular fibrillation.
\end{abstract}

Key words: J wave

(Int Heart J 2019; 60: 185-188)

$\mathrm{F}$ or decades, an early repolarization (ER) pattern, defined as notches or slurs in the terminal portion of the QRS complex (J-wave), has been considered to be a benign ECG finding. However, in recent years, robust population-based studies have established an association between $\mathrm{J}$-waves and ventricular fibrillation (VF), whether idiopathic in nature or secondary to ischemi. ${ }^{1-3)}$ Also, in patients presenting with aborted cardiac arrest who display ER in the inferior and/or lateral leads, documented VF is generally diagnosed as ER syndrome (ERS). ${ }^{4,5)}$

Therapeutic hypothermia is a standard strategy to improve neurological outcome in comatose patients after cardiac arrest. $\left.{ }^{6}\right)$ J-waves are an established ECG manifestation of hypothermia, ${ }^{7-10)}$ however, data linking hypothermic J-waves and VF are sparse.

We present here a patient with ERS who survived from a VF episode. In this patient, on admission, no Jwaves could be recognized due to the presence of sinus tachycardia and slowing of the patient's heart rate unmasked the J-waves. Therapeutic hypothermia exacerbated the J-wave amplitude and morphology, resulting in refractory VF episodes.

\section{Case Report}

A 42-year-old man with paroxysmal atrial fibrillation suffered an out-of-hospital cardiac arrest at a convenience store in the morning. Following immediate cardiopulmonary resuscitation and successful defibrillation of the VF using an automated external defibrillator, he was transferred to our hospital. On his arrival at our emergency room, his consciousness level was 5 points on the Glas- gow Coma Scale (eye, verbal, and motor scores were 1, 1, and 3, respectively). He was taking disopyramide (300 $\mathrm{mg}$ per day) and a $\beta$-blocker (10 mg of carvedilol per day) for paroxysmal atrial fibrillation, but the disopyramide was halted two days before admission. He had no other past medical history or specific family history. The ECG on admission exhibited sinus tachycardia (120 beats/minutes [bpm]) with no J-waves (Figure 1). The plasma electrolyte levels were normal, and transthoracic echocardiography revealed a normal ventricular function without any significant abnormalities. Emergent coronary angiography revealed normal coronary arteries. Hypothermia therapy was performed in the intensive care unit. Approximately 5 hours later, at a body temperature (BT) of the patient of $33.5^{\circ} \mathrm{C}$, VF occurred (Figure 2E). The VF was finally defibrillated by an eighth shock with a direct current of $270 \mathrm{~J}$ and converted to atrial fibrillation (Figure 2 F). After the VF was defibrillated, we finally noticed the presence of J-waves during hypothermia and halted the hypothermia therapy.

The 12-lead ECGs and ECG monitor recordings before the VF episodes were retrospectively evaluated, and the changes in the J-wave amplitude and morphology after stopping the hypothermia therapy were prospectively observed. Fifteen minutes after beginning the hypothermia with a heart rate of $96 \mathrm{bpm}$ and $\mathrm{BT}$ of $36.8^{\circ} \mathrm{C}$, a slurred J-wave with an amplitude of $4 \mathrm{~mm}$ appeared on the ECG monitoring (Figure 2A). The J-wave amplitude gradually became augmented, and its morphology changed from a "slurred" type to a "notched" type parallel with a decrease in the BT (Figure 2B-D). Just before the VF occurrence, the notched J-wave amplitude was $12 \mathrm{~mm}$ (Figure 2E). Just after a successful VF defibrillation (Figure 2F), and

From the ${ }^{1}$ Department of Cardiovascular Medicine, Faculty of Medical Sciences, University of Fukui, Fukui, Japan.

Address for correspondence: Shinsuke Miyazaki, MD, Department of Cardiovascular Medicine, Faculty of Medical Sciences, University of Fukui, 23-3 Matsuokashimoaizuki, Eiheiji-cho, Yoshida-gun, Fukui 910-1193, Japan. E-mail: mshinsuke@k3.dion.ne.jp

Received for publication January 11, 2018. Revised and accepted April 23, 2018.

Released in advance online on J-STAGE November 20, 2018.

doi: 10.1536/ihj.18-024

All rights reserved by the International Heart Journal Association. 


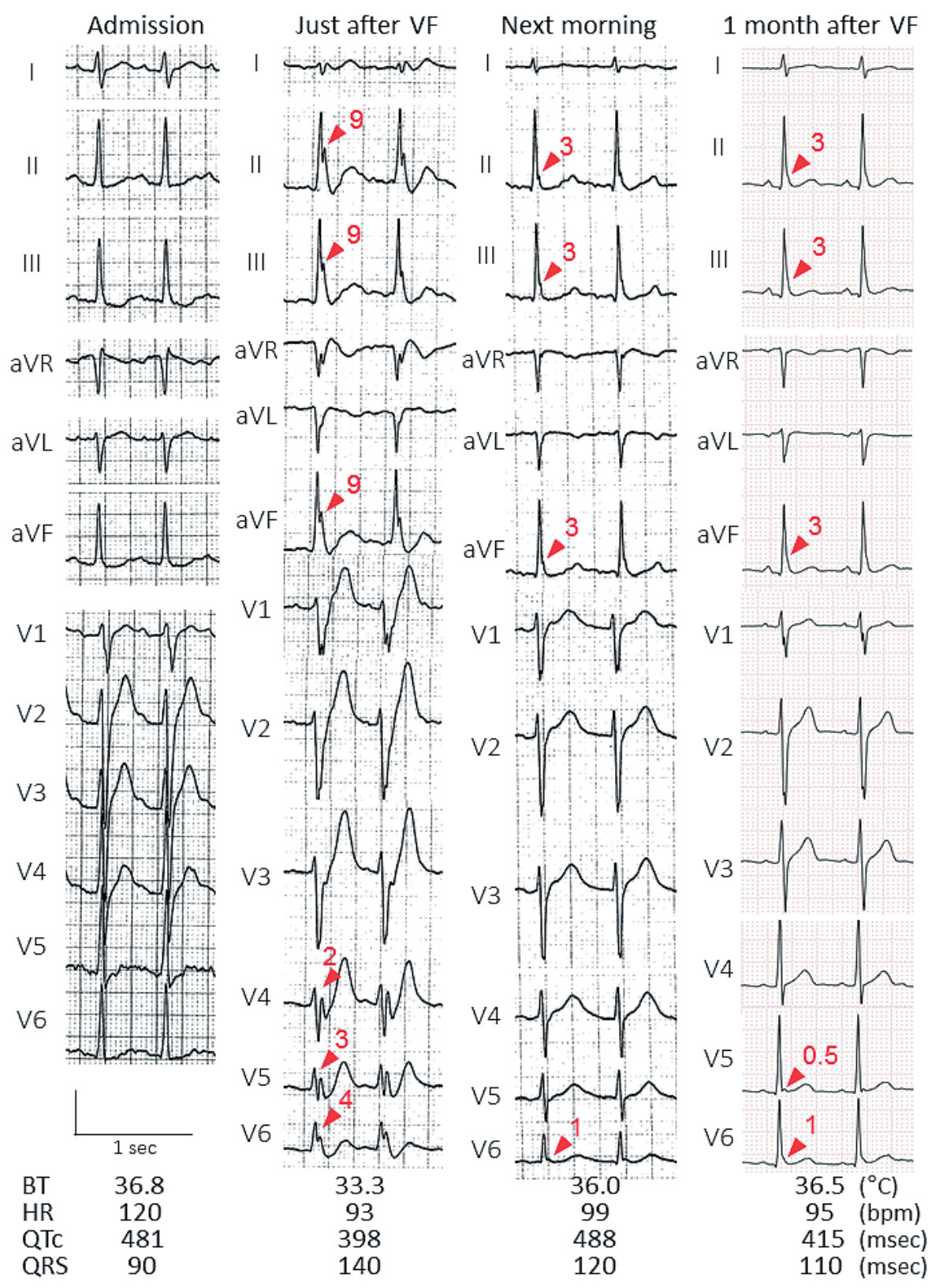

Figure 1. The 12-lead electrocardiograms on admission, just after a successful shock for VF, the next day, and at discharge ( 1 month after VF). The numbers in the image indicate the height of the $\mathrm{J}$ waves (mm). The body temperature (BT), heart rate (HR), and corrected QT Interval (QTc) at each time point are presented at the bottom. bpm indicates beat per minutes.

on cessation of the hypothermia, BT was $33.1^{\circ} \mathrm{C}$ and heart rate was $55 \mathrm{bpm}$ with high amplitude $(8 \mathrm{~mm}) \mathrm{J}$-waves (Figure 2G). Therefore, during the rewarming phase, we started an isoproterenol infusion at $0.01 \mu \mathrm{g} / \mathrm{min} / \mathrm{kg}$. The heart rate gradually increased and the amplitude of the $\mathrm{J}$ waves gradually attenuated with the morphology change from a "notched" type to a "slurred" type parallel with rewarming of the BT (Figure 2G-I). On the next morning, notched $\mathrm{J}$-waves were present in the infero-lateral leads (Figure 1). The patient was extubated without any neurological deficits, and the isoproterenol infusion was halted.
The late potentials were negative on the signalaveraged ECG, and no late gadolinium enhancement was found by cardiac magnetic resonance imaging (MRI). No abnormal findings were detected on the fluorodeoxyglucose positron emission tomography or ${ }^{123} \mathrm{I}-15$-(piodophenyl)-3(R,S)-methylpentadecanoic acid scintigraphy. A genetic analysis of several genes previously implicated in repolarization disorders did not identify any putative disease causing the mutations. The patient underwent catheter ablation of atrial fibrillation, and received an implantable cardioverter defibrillator. One month later, the 
A. $11: 00$ BT $36.8^{\circ} \mathrm{C}, \mathrm{HR} 96 \mathrm{bpm}, \mathrm{QRS} 100 \mathrm{~ms}$

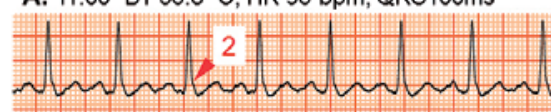

B. $14: 00$ BT $35.7^{\circ} \mathrm{C}$, HR $84 \mathrm{bpm}$, QRS $120 \mathrm{~ms}$

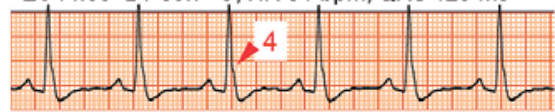

C. $14: 15$ BT $35.0^{\circ} \mathrm{C}, \mathrm{HR} 79 \mathrm{bpm}, \mathrm{QRS} 140 \mathrm{~ms}$
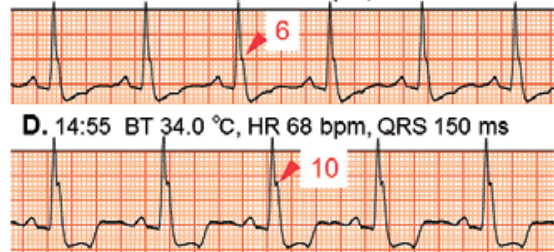

E. $15: 25$ BT $33.5{ }^{\circ} \mathrm{C}, \mathrm{HR} 64 \mathrm{bpm}, \mathrm{QRS} 160 \mathrm{~ms}$

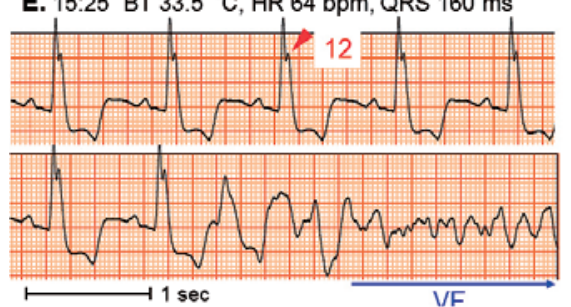

F. $12: 25$ BT $33.5^{\circ} \mathrm{C}, \mathrm{HR} 64 \mathrm{bpm}$,

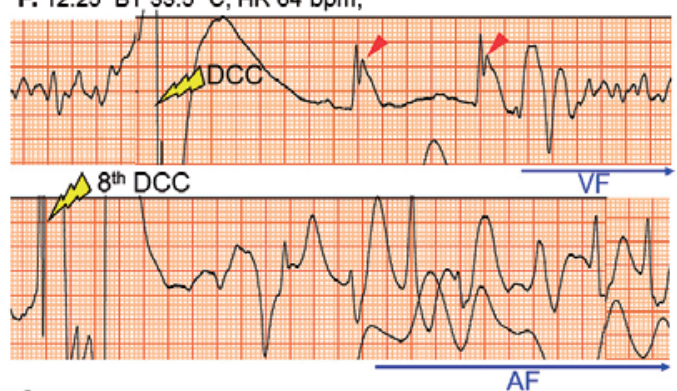

G. $17: 00 \mathrm{BT} 33.1^{\circ} \mathrm{C}, \mathrm{HR} 55 \mathrm{bpm}, \mathrm{QRS} 160 \mathrm{~ms}$

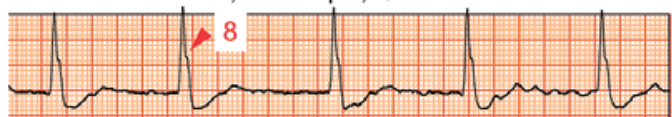

H. $19: 10$ BT $34.0^{\circ} \mathrm{C}, \mathrm{HR} 78 \mathrm{bpm}$, QRS $120 \mathrm{~ms}$

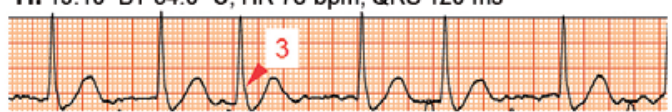

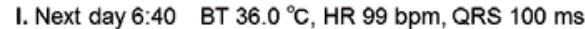

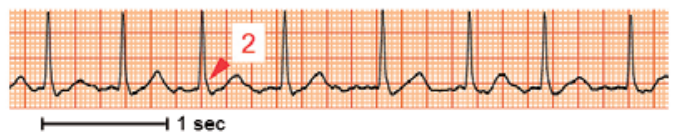

Figure 2. The time-course of a bedside continuous single lead ECG monitoring during therapeutic hypothermia A-E: Cooling phase; F: Direct current cardioversion [DCC] of VFs; G-I: Rewarming phase. The numbers in the image indicate the height of the $\mathrm{J}$ waves $(\mathrm{mm})$. The time, body temperature, and heart rate are presented on the top of each tracing. AF indicates atrial fibrillation; $\mathrm{HR}$, heart rate; and VF, ventricular fibrillation

patient was discharged without the use of any antiarrhythmic drugs, but "slurred" J-waves were still present in the infero-lateral leads on the 12-lead ECG (Figure 1). To date, the patient has done well with no VF episodes during a 17-month follow-up.

\section{Discussion}

In the present case, J-waves were absent with a heart rate of 120 beats per minutes on admission. However, shortly after starting therapeutic hypothermia with a slower heart rate, slurred J-waves appeared. With a decreasing BT, the J-wave amplitude became augmented with a change in the J-wave morphology from slurring to notching, and, finally, VF occurred. During the rewarming phase, both the amplitude and distribution of the J-waves in the infero-lateral leads became attenuated with an increasing BT. The J-waves were still present in the inferior leads upon discharge, but no VF occurred during his follow up.

Several studies have shown that a bradycardiadependent augmentation of J-waves is often found in ERS patients with VF episodes. ${ }^{3,11}$ A recent study ${ }^{12}$ showed that, at a higher rate, J-waves become attenuated in ERS patients whereas they are augmented in ER subjects with no VF episodes. Thus, an inverse relationship between the $\mathrm{J}$-wave amplitude and heart rate is a characteristic of ERS. In the present case, J-waves were absent with a heart rate of 120 beats per minutes on admission, and we did not recognize that the present case had ERS. However, at the beginning of the therapeutic hypothermia and with a slower heart rate, J-waves in the infero-lateral leads appeared. On emergency visits shortly after cardiopulmonary resuscitation, patients may often have tachycardias, as was the case in this research. In such a setting, and with no Jwaves on the 12-lead ECGs, there is a fair possibility that physicians will not be able to recognize the presence of idiopathic VF. Careful and frequent recording and evaluation of the 12-lead ECGs are indispensable so as not to miss any underlying ERS.

To improve the neurological status, the guidelines $\left.{ }^{6}\right)$ advise performing induced hypothermia $\left(32^{\circ} \mathrm{C}\right.$ to $34^{\circ} \mathrm{C}$ ) for a sub group of patients with out-of-hospital VF episodes after cardiac arrest and for most other comatose patients after a cardiac arrest. ${ }^{6}$ The precise duration and optimal temperature targets are unknown, but 12 to 24 hours duration at temperature of $32^{\circ} \mathrm{C}$ to $34^{\circ} \mathrm{C}$ are recommended based on the regimens studied in prior trials. ${ }^{6}$ Although hypothermia is known to result in J-wave augmentation, ${ }^{7-10)}$ there seems to be less evidence that the temporal development and augmentation of J-waves during therapeutic hypothermia results in VF. A previous study prospectively examined the ECG changes during therapeutic hypothermia in 47 patients. ${ }^{9)}$ Although J-waves appeared in $21.3 \%$ of these patients and the amplitude and duration of the J-waves correlated with the temperature, none of the patients experienced any life-threatening arrhythmias. In another study ${ }^{10)}$ with 43 survivors of cardiac arrest, which includes patients with idiopathic VF and coronary artery disease-related VF, hypothermia increased both the prevalence and magnitude of the J-waves in these survivors. However, no patients exhibited any VF during 
the therapeutic hypothermia either. Thus, there have been a few cases in which J-point augmentation during hypothermia has resulted in VF in patients with idiopathic VF, not Brugada syndrome. ${ }^{13,14)}$ In this case, sinus tachycardia masked the J-waves, and therapeutic hypothermia exaggerated the J-waves and triggered VF attacks. Continuous ECG monitoring disclosed that therapeutic hypothermia augmented the $\mathrm{J}$-waves with changes in the $\mathrm{J}$ waves morphology from slurring to notching, in addition to a decreased heart rate and prolonged QRS width, and that, finally, VF could occur. These ECG changes in addition to the lack of late potentials might support repolarization as the main mechanism of J-wave occurrence. If more careful attention had been paid to the ECG changes, we could have avoided the occurrences of VF. Although Jwaves can be observed on ECG monitoring, bedside continuous single-lead ECG monitoring might be insufficient to recognize the appearance of J-waves in the inferolateral leads.

Several ERS cases who survived from an aborted death or VF have been reported. ${ }^{15)}$ However, to the best of our knowledge, there have been no reports in which the detailed and serial changes in the J-wave amplitude and morphology during therapeutic hypothermia and occurrences of VF were recorded. Therapeutic hypothermia can lead to VF due to an exacerbation of J-waves. This seems to be generally rare, but should be noted as an adverse event during the global use of therapeutic hypothermia in patients with cardiac arrest. During acute care in patients shortly after an aborted cardiac arrest or documented VF, careful and frequent recording and evaluation of the 12lead ECGs and/or careful observation of the ECG monitor are indispensable, so as not to miss any underlying ERS.

\section{Acknowledgments}

We thank Mr. John Martin for his help in the preparation of the manuscript.

\section{Disclosures}

Conflicts of interest: None declared.

\section{References}

1. Haïssaguerre M, Derval N, Sacher F, et al. Sudden cardiac arrest associated with early repolarization. N Engl J Med 2008; 358: 2016-23.

2. Tikkanen JT, Anttonen O, Junttila MJ, et al. Long-term outcome associated with early repolarization on electrocardiography. $\mathrm{N}$ Engl J Med 2009; 361: 2529-37.

3. Nam GB, Kim YH, Antzelevitch C. Augmentation of J waves and electrical storms in patients with early repolarization. $\mathrm{N}$ Engl J Med 2008; 358: 2078-9.

4. Miyazaki S, Shah AJ, Haïssaguerre M. Early repolarization syndrome - a new electrical disorder associated with sudden cardiac death. Circ J 2010; 74: 2039-44.

5. Antzelevitch C, Yan GX, Ackerman MJ, et al. J-Wave syndromes expert consensus conference report: Emerging concepts and gaps in knowledge. J Arrhythm 2016; 32: 315-39.

6. Callaway CW, Donnino MW, Fink EL, et al. Part 8: PostCardiac Arrest Care: 2015 American Heart Association Guidelines Update for Cardiopulmonary Resuscitation and Emergency Cardiovascular Care. Circulation 2015; 132(18 Suppl 2): S46582.

7. Osborn JJ. Experimental hypothermia; respiratory and blood $\mathrm{pH}$ changes in relation to cardiac function. Am J Physiol 1953; 175: $389-98$

8. Tomaszewski W. Changements électrocardiographiques observés chez un homme mort de froit. Arch Mal Coeur 1938; 31: 525-8.

9. Salinas P, Lopez-de-Sa E, Pena-Conde L, et al. Electrocardiographic changes during induced therapeutic hypothermia in comatose survivors after cardiac arrest. World J Cardiol 2015; 7: 423-30.

10. Williams SE, Sabir I, Nimmo C, et al. Quantitative assessment of the effects of therapeutic hypothermia on early repolarization in idiopathic ventricular fibrillation survivors: a 7-year cohort study. Circ Arrhythm Electrophysiol 2014; 7: 120-6.

11. Aizawa Y, Sato A, Watanabe H, et al. Dynamicity of the J-wave in idiopathic ventricular fibrillation with a special reference to pause-dependent augmentation of the J-wave. J Am Coll Cardiol 2012; 59: 1948-53.

12. Aizawa Y, Takatsuki S, Nishiyama T, et al. Tachycardia-induced $\mathrm{J}$-wave changes in patients with and without idiopathic ventricular fibrillation. Circ Arrhythm Electrophysiol 2017; 10: e005214.

13. Bastiaenen R, Hedley PL, Christiansen M, Behr ER. Therapeutic hypothermia and ventricular fibrillation storm in early repolarization syndrome. Heart Rhythm 2010; 7: 832-4.

14. Federman NJ, Mechulan A, Klein GJ, Krahn AD. Ventricular fibrillation induced by spontaneous hypothermia in a patient with early repolarization syndrome. J Cardiovasc Electrophysiol 2013; 24: 586-8.

15. Hwang KW, Nam GB, Han J, et al. Incidence of atrial tachyarrhythmias in patients with early repolarization syndrome. Int Heart J 2017; 58: 43-9. 